\title{
Angiomatous nasal polyp: Clinical diagnostic dilemma
}

\author{
Sunder Goyal1, Ghanshyam Jayavardhan², Snigdha Goyal'3, Isha Saini ${ }^{4}$ \\ ${ }^{I}$ Department of General and Minimal Invasive Surgery, Kalpana Chawla Govt. Medical College, Karnal, India \\ ${ }^{2}$ Department of Ear Nose and Throat (ENT), Kalpana Chawla Govt. Medical College, Karnal, India \\ ${ }^{3}$ Department of Pathology, Dr. RML Postgraduate Institute of Medical Sciences, New Delhi, India \\ ${ }^{4}$ Life Line Clinical \& Diagnostic Centre, Karnal, Haryana, India
}

Received October 12, 2014; Revised January 06, 2015; Accepted January 08, 2015; Published Online January 10, 2015

\section{Case Report}

\begin{abstract}
Angiomatous polyp (Angiectatic nasal polyps) is rare and its incidence is $4-5 \%$ of all nasal polyps. As it occurs with variable clinical features and there is no confirmatory preoperative investigation, clinical diagnosis can be a dilemma. Clinical picture of angiofibroma, simple antrochoanal polyp and inverted papilloma may resemble with each other. As polyps invade surrounding bone, these should be distinguished from a malignant mass. We present an interesting case of an infarcted angiectatic nasal polyp with extensive surrounding bony destruction. Correct preoperative radiological diagnosis is important to avoid unnecessary extensive surgery. Histopathological evaluation of polyps is mandatory since they require different treatment due to difference in the prognosis.
\end{abstract}

Keywords: Angiomatous Polyp; Bone Destruction; Benign

\section{Introduction}

The angiectatic polyps (ANP) account for only $4-5 \%$ of inflammatory nasal polyps ${ }^{1}$ and have variable clinical features and growth patterns. ${ }^{1,2}$ It is also called nasal polyp with hemorrhage and necrosis. In ANPs, there is prominent component of dilated capillary-type blood vessels. ${ }^{1}$ The polyp gets infarcted due to necrosis as the result of widespread intraluminal thrombosis. ${ }^{2}$ These polyps can cause extensive bone destruction due to local aggressiveness and can resemble a neoplastic process and should be distinguished from malignancy. ${ }^{3}$ These cases present with variable clinical features and mimics with other pathologies including malignancy. Few cases have been reported in literature. ${ }^{4}$ In this report, we present a case of benign angiomatous nasal polyp with local bony destruction.

\section{Case Report}

A 43-year-old female presented with 7 month old history of right nasal obstruction, headache, epiphora, anosmia, and swelling below the medial canthus of right eye. She complained of epistaxis and blood stained nasal discharge. She denied any visual disturbance. She took ayurvedic and homeopathic treatment without any relief.

During the examination, a grey white polypoidal mass was noticed. It was with irregular ulcerated surface with hemorrhagic areas filling the whole right nasal cavity. Mass was pushing the septum to left and thus causing her medial can- thus to bulge. A probe could be passed all around the mass except the lateral wall. Bleeding occurred on probing.

There was a large heterogeneously enhancing soft tissue mass of size $6.3 \times 6.7 \times 6.1 \mathrm{~cm}$ in right sinonasal cavity (Figure 1).

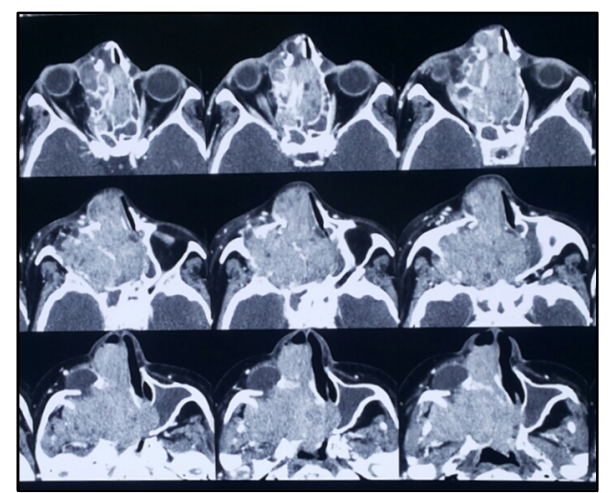

FIG. 1: Axial view of enhanced computed tomography

Mass was extending superiorly into ethmoid sinus with destruction of septa. Bulging of medial wall of left orbit was seen. Mass was extending laterally into masticator space with erosion of lateral wall of maxillary sinus. Mass was extending posteriorly also into right pterygoid plates with erosion of right medial pterygoid plate. Mass extended into the infratemporal fossa through pterygomaxillary fissure (Figure 2). This was suggestive of a neoplastic etiology. 
Small biopsy sent for primary morphological diagnosis revealed benign sinonasal polyp with prominent squamous metaplasia and oncytic epithelium with senescent atypia changes. Mass was excised almost completely through the transnasal route using $0^{\circ}$ and $45^{\circ}$ nasoendoscopes. The mass was seen arising from right maxillary antrum, enlarging the ostium, extending superiorly to anterior ethmoidal complex and posteriorly to nasopharynx. Bone erosion, destruction of maxilla and involvement of the infratemporal fossa through pterygomaxillary fissure was a unique feature. Significant amount of bleeding occurred intra operatively which required 3 units of blood transfusion. Postoperative period was uneventful.

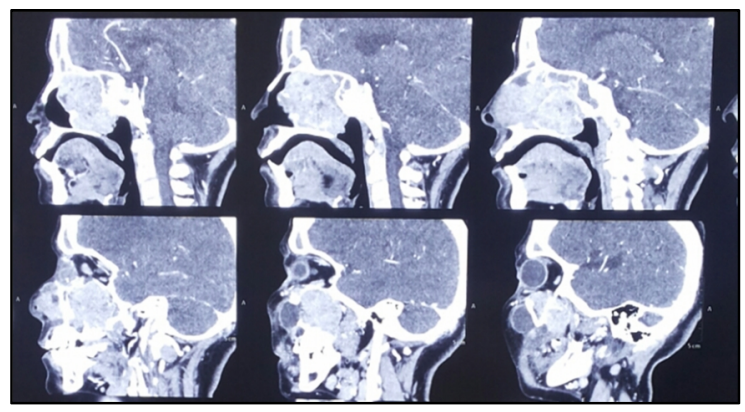

FIG.2: Sagittal view of enhanced computed tomography

Multiple grey white irregular tissue pieces were excised. Grey brown polypoidal mass measured $3 \times 2 \times 1 \mathrm{~cm}$ and $3 \times 1$ $\times 1 \mathrm{~cm}$ respectively. Histopathological examination revealed benign sinonasal polyposis with focal squamous metaplasia and mild dysplasia. No evidence of malignancy.

Post operatively the patient improved. The swelling on the medial canthus of right eye disappeared. The patient was discharged after 7 days. No sign of recurrence after 3 Months postoperative follow up.

\section{Discussion}

Depending upon the basis of predominant stromal eleme nt, inflammatory sinonasal polyps are classified into five types: edematous, glandular, fibrous, cystic and angiomatous/angiectatic polyp. Mostly, they present as soft, gelatinous translucent polypoidal, painless mass with nasal discharge. They result in gradual obstruction of the nasal cavity. Other presenting clinical symptoms are loss of smell sensation, epistaxis, exophthalmoses, proptosis and visual disturbances. ANPs may be a variant of sinonasal polyps and are usually derived from antrochoanal polyps. Their vascular supply is proneto compression. ${ }^{2}$

It is hypothesized that initial vascular dilatation/ectasia, extravascular edema and possibly infarction develop due to vascular compromise. There may be reactive and reparative changes with neo-vascularisation and result in formation of a polyp. Angiectatic nasal polyps are considered to be a variant of antrochoanal polyp. ${ }^{5}$ Predominant features of infarcted ANPs are clusters of ectatic blood vessels surrounded by abundant fibrin-like eosinophilic extracellular material ${ }^{4}$ and superimposed fibrinoid necrosis, luminal thrombosis of ectatic blood vessels.1, 2,3,6 These polyps can grow very fast and thus result in bone erosion that could mimic malignancy clinically and thus result in diagnostic dilemma. ${ }^{3}$

In angiectatic polyps, scattered atypical pleomorphic spindle cells (Myofibroblasts) in the stroma are quite common whereas less common in sinonasal polyp., ${ }^{1,7}$ Non epithelial tumors of the nasal cavity and nasopharynx are usually vascular tumours. ${ }^{8}$ Due to prominent vascular component of ANPs, there is diagnostic dilemma mainly with capillary or cavernous hemangioma ${ }^{9}$ and nasopharyngeal angiofibroma. ${ }^{10}$

The vascular lamina of cavernous hemangioma is usually larger than those of angiomatous polyp. ${ }^{6}$ Histomorphology, age, sex, and site helps in differentiation. Angiofibroma occurs in young males, whereas sinonasal angioma do not show age or sex predilection. Sinonsal angiomas occur more often in the anterior nasal septum, the turbinate and vestibule. The presenting symptoms are common in both diseases.

Correct diagnosis is necessary, as they require diinoid necrosis, luminal thrombosis of ectatic blood vessels. ${ }^{1,2,3,6}$ These polyps can grow rapidly, causing bone erosion that could simulate malignancy preoperatively. ${ }^{3}$ Scattered atypical pleomorphic spindle cells (Myofibroblasts) in the stroma are part of reactive secondary changes are common feature of angiectatic polyps and rarely seen in sinonasal polyp. ${ }^{1,7}$ However, other vascularised fibromatous angiomatous polypsdo not show deposition of pseudoamyloid- like eosinophilic material, superimposed fibrinoid thrombosis and fibrinoid necrosis of blood vessels wall, or pseudosarcomatous stroma.

Accurate diagnosis is mandatory since management and prognosis are different in both the conditions. It is problematic to differentiate angiomatous polyp from juvenile angiofibroma clinically and may need to rely on imaging features and pattern of growth for distinction between these two entities. On CT scans, angiomatous polyps appear as non-enhancing or minimally enhancing nasal vault masses without pterygopalatine fossa involvement. Whereas angiofibroma has typical hypervascular appearance in contrast to angiomatous. Polyp and usually extend the through the roof of nasopharynx into the sphenoidal sinus and pterygopalatine fossa. Rarely ANPS can also invade into sphenoidal sinus ${ }^{11}$ and ethmoidal sinus. ${ }^{6}$

Conventional magnetic resonance imaging (MRI) is a better modality for preoperative diagnosis of the angiomatous nasal polyp, and show characteristic hypointensity on T1 weighted images and internal heterogeneous hyperintensity with a peripheral hypointense rim on T2 weighted images, as well 
as and strong nodular and patchy enhancement on postcontrast MRIs. ${ }^{2,9,11}$ Moreover, progressive enhancement on DCE MRI is very important diagnostic clue. ${ }^{9}$ Areas of mixed signal intensity on T2 weighted images are supposed to be caused by the extensive areas of organized thrombus and necrosis in that part of polyp and the peripheral hypointense rim on $\mathrm{T} 2$ weighted images due to old microhemorrhage with hemosiderin deposition on the surface of the polyp. ${ }^{2}$ Post contrast strong enhancement of nasochoanal portion of ANP suggest extensive vascular proliferation and ectasis. ${ }^{2}$

Angiography may be used for early diagnosis and to differentiate ANP from juvenile angiofibroma. ${ }^{2}$ ANPs show hypovascular or avascular appearance on angiography due to their irregular racemose arrangements of dilated capillary-type vessels, in contrast to normal arborizing pattern of vascularity. ${ }^{11}$ Specific locations of angiofibroma in pterygopalatine fossa with absent flow voids on MRI can have characteristic histologic features of stellate and staghorn blood vessels set in. Compression by cellular fibroblastic stroma differentiates it from ANPs, which have racemose aggregates of irregularly shaped dilated capillary-like blood vessels. ${ }^{1,2}$ However, correct diagnosis can be based on its anatomic location and association with inflammatory edematous sinonasal polyp. ${ }^{2}$ If there is pseudoepitheliomatous hyperplasia of the surface epithelium of ANP then it should be differentiated from squamous cell carcinoma and histopathology is mandatory to prove the diagnosis.

Most ANPs arise in maxillary sinus and extend towards the choana and into the nasopharynx ${ }^{2,9}$ and the most common symptoms are nasal obstruction and recurrent epistaxis. Gradual enlargement of the lesion may cause erosion, displacement of the adjacent bony structures, cheek swelling, and exophthalmos to manifest. ${ }^{6}$ There are no clear guidelines for management; however, the transnasal endoscopic surgical excision continues to be the treatment of choice. Prognosis is good and recurrence is rare. ${ }^{4}$

\section{Conclusion}

Angiectatic polyps present with atypical features. ANPs are benign and may behave more aggressively than other angiomatous polyps and may mimic malignancy. Hence, alertness of their presence and morphology is very significant for proper management.

\section{Conflict of interest}

The authors declare that they have no conflicts of interest. The authors alone are responsible for the content and writing of the paper.

\section{References}

1. Yfantis HG, Drachenberg CB, Gray W, Papadimitriou JC. Angiectatic nasal polyps that clinically simulate a malignant process: report of 2 cases and review of the literature. Arch Pathol Lab Med 2000; 124:406-10.

2. Sheahan P, Crotty PL, Hamilton S, et al. Infarcted angiomatous nasal polyps. Eur Arch Otorhinolaryngol 2005; 262: 225-30.

3. Kim JY, Nam SY, Kwon SJ, Jun SY. Angiomatous nasal polyp with maxillary wall erosion. J Rhinol 2007; 14: 125-7.

4. Jayaram S, Svecova N, Biggs TC, Theaker J, Salib RJ. Angiectatic Nasal Polyp - The Great Imitator. The Internet Journal of Otorhinolaryngology 2013; 15.

5. Dai LB, Zhou SH, Ruan LX, Zheng ZJ. Correlation of computed tomography with pathological features in angiomatous nasal polyps. PLoS One 2012; 7:e53306.

6. Kim EY, Kim HJ, Chung SK, et al. Sinonasal organized hematoma: CT and MR imaging findings. AJNR Am J Neuroradiol 2008; 29:1204-8.

7. Tuziak T, Kram A, Woyke S. Edematous nasal polyp with atypical stromal cells misdiagnosed cytologically as rhabdomyosarcoma. A case report. Acta Cytol 1995; 39:521-4.

8. Fu YS, Perzin KH. Non-epithelial tumors of the nasal cavity, paranasal sinuses, and nasopharynx: A clinicopathologic study. I. General features and vascular tumors. Cancer 1974; 33:1275-88.

9. Wang YZ, Yang BT, Wang ZC, et al. MR evaluation of sinonasal angiomatous polyp. AJNR Am J Neuroradiol 2012; 33:767-72.

10. Nota J, Hinohira Y, Takahashi H, et al. Angiectatic nasal polyp localized in maxillary sinus. Japanese Journal of Rhinology 2008; 47: 105-8.

11. De Vuysere S, Hermans R, Marchal G. Sinonasal polyp and its variant, the angiomatous polyp : MRI findings. Eur Radiol 2001; 1: 55-8. 